\title{
Simple Coalitional Strategy Profiles in Repeated Games*
}

\author{
C. LARREA ${ }^{\dagger}$ and L. RUIZ
}

\begin{abstract}
In this paper we introduce simple coalitional profiles to avoid group deviations in repeated games. In the repeated Cournot supergame we prove that it is possible to sustain the symmetric monopoly outcome by means of a variety of strategies which satisfy the requirement that no coalition (other than the grand coalition) will deviate in any subgame.
\end{abstract}

JEL classification: C70; C71; C72

Keywords: Repeated games, coalitional strategy profiles

${ }^{*}$ This work is financially supported by the Spanish Government ( ECO2012-31346) and by the Basque Government (IT568-13 and IT869-13). We thank to J. Albizuri and E. Iñarra for useful comments.

${ }^{\dagger}$ Dept. Economía Aplicada IV, BRiDGE, University of the Basque Country (UPV/EHU). Email: mariaconcepcion.larrea@ehu.es

${ }^{\ddagger}$ Dept. Economía Aplicada IV, University of the Basque Country (UPV/EHU). Email: luismariano.ruiz@ehu.es 


\section{Introduction}

Most of the equilibrium concepts in the literature of infinitely repeated games may suffer from a serious drawback: they do not consider the possibility of a group of players forming a coalition to deviate. Subgame perfect equilibrium strategies are defined to avoid single player deviations. Deviations of two or more players are often ignored. In a ground-breaking paper, Horniacek (1996) argues that group deviations should not be ignored, and that any deviation of any coalition (other than the grand one) must be punished by the complementary coalition. Interested readers are referred to Horniacek's paper (1996, pp. 101-102).

In order to punish coalitional deviations we introduce simple coalitional strategy profiles which generalize the simple strategy profiles defined by Abreu (1989). A simple coalitional strategy profile consists of one cooperative path and one punishment path for each coalition other than the grand one. These strategies are defined as follows: Start the cooperative path and remain on it if no player deviates. If, a coalition deviates after any history, then start the punishment phase of that coalition. Only deviations of all players are ignored.

The equilibrium concept used throughout the paper is the Quasi Strong Perfect Equilibrium (QSPE) introduced by Horniacek (1996). An equilibrium is QSPE if no coalition can, taking the actions of its complement as given, deviate in a way that benefits all of its members. It is explained in Section 3 why the Strong Perfect Equilibrium of Rubinstein (1980) cannot be used.

Next we outline why the problem of checking whether a simple coalitional strategy profile is QSPE can be so complex especially when the number of players $n$ is big. To avoid coalitional deviations we need to punish all coalitions except the grand one. Even in the simplest case of a single punishment for each coalition (irrespective of the phase in which the deviation has taken place) deviations of any of the $2^{n}-2$ coalitions from any of the $2^{n}-1$ outcome paths must be avoided. Each coalition could deviate for only one period, for any finite number of periods, or even forever. Furthermore, coordinated deviations (which are explained in detail in Section 3) must be taken into account. As will be shown, these coordinated deviations could potentially be infinitely complex. 
A relevant contribution of this paper is to simplify this problem substantially. In Section 3 we generalize a result similar to that of Abreu in (1989). We prove that only one-shot deviations need to be checked to avoid coalitional deviations, where a one-shot deviation is a single-period deviation followed by sticking to the strategy in subsequent periods.

To obtain all the major results of this paper we need to introduce an auxiliary equilibrium concept which is even stronger than QSPE, and which we call the Quasi Even Stronger Perfect Equilibrium (QESPE). An equilibrium is QESPE if no coalition other than the grand one, taking the actions of its complement as given, can deviate in a way that increases the sum of the payoffs of all of its members. Note that if a strategy is QESPE then it is also QSPE, whereas the reverse is not true.

One of the contributions of this paper is to show that in the Cournot supergame with any number of players it is possible to sustain the symmetric monopoly outcome by means of a variety of strategies which satisfy the requirement that no coalition other than the grand one may deviate in any subgame (provided that the discount factor is close enough to 1). A straightforward conclusion from this result is that, at least in the symmetric Cournot model, any coalition which has the possibility of improving the payoffs of all of its members with a deviation, also has different strategies for sustaining that deviation in a credible way (where credibility means that no subcoalition will deviate further). This enables us to conclude that any deviation of any coalition must be punished by the complementary coalition.

The rest of the paper is organized as follows. Section 2 contains the preliminaries. Section 3 and 4 present the results. Section 5 concludes with some comments on related work, with special attention to Horniacek (1996). Appendix is divided into two parts, one with the lemmas used in the paper and their proofs and the other with the proofs of the results.

\section{Preliminaries}

Let $G=\left(Q_{1}, \ldots, Q_{n} ; \Pi_{1}, \ldots, \Pi_{n}\right)$ be an n-player game where $N=\{1, \ldots, n\}$ is the set of players, $Q_{i}$ is the set of actions $q_{i}$ of player $i$ and $\Pi_{i}: Q=$ $Q_{1} \times \ldots \times Q_{n} \longrightarrow R$ is player $i$ 's payoff function.

The associated infinitely repeated game with discounting is denoted by 
$G^{\infty}(\delta)$ where $\delta \in(0,1)$ is the discount factor. If $q(t)=\left(q_{1}(t), \ldots, q_{n}(t)\right)$ is the vector of actions played in period $t$, then $\{q(1), \ldots, q(t)\}$ is a history $h$ of length $t$. A strategy $\sigma_{i}$ of player $i$ in $G^{\infty}(\delta)$ is a sequence of functions $\sigma_{i}^{t}$ (or $\sigma_{i}(t)$ ) from the set of all histories of length $t-1$ to $Q_{i}$, so $\sigma_{i}^{1} \in Q_{i}$ is the initial action of player $i$. A stream of action profiles $\{q(t)\}_{t=1}^{\infty}$ is referred to as an outcome path and is denoted by $S$. A strategy profile $\sigma=\left(\sigma_{i}\right)_{i \in N}$ generates an outcome path $S(\sigma)=\{q(\sigma)(t)\}_{t=1}^{\infty}$ defined inductively by:

$$
\begin{aligned}
& q(\sigma)(1)=\sigma^{1} \\
& q(\sigma)(t)=\sigma^{t}(q(\sigma)(1), \ldots, q(\sigma)(t-1)), \text { if } t>1 .
\end{aligned}
$$

The value $\Pi_{i}(q(t))$ denotes the payoff of player $i$ in period $t$ when the outcome in this period is $q(t)$. And $\Pi_{i}(S)$ denotes the discounted payoff of player $i$ for the outcome path $S=\{q(t)\}_{t=1}^{\infty}$ :

$$
\Pi_{i}(S)=\sum_{t=1}^{\infty} \delta^{t-1} \Pi_{i}(q(t)) .
$$

Then, the discounted payoff of player $i$ in $G^{\infty}(\delta)$ obtained with the strategy profile $\sigma$ is $\Pi_{i}(\sigma)=\Pi_{i}(S(\sigma))^{1}$.

$\Pi_{i}(\sigma \mid h)$ denote the discounted payoff of player $i$ when $(\sigma \mid h)$ is the continuation of $\sigma$ after $h$.

A coalition $D$ is a nonempty subset of $N$. Let $Q_{D}=\prod_{i \in D} Q_{i}, Q_{-D}=$ $\prod_{j \in N \backslash D} Q_{j}, q_{D}=\left(q_{i}\right)_{i \in D} \in Q_{D}$ and $q_{-D}=\left(q_{j}\right)_{j \in N \backslash D} \in Q_{-D}$. We denote by $\sigma_{D}=\left(\sigma_{i}\right)_{i \in D}$ a strategy of coalition $D$ and by $\sigma_{-D}=\left(\sigma_{j}\right)_{j \in N \backslash D}$.

In this paper, we consider the Cournot Supergame with perfect monitoring. So the preliminaries for this model are also introduced here.

Take $n$ firms producing a homogeneous product at a constant marginal $\operatorname{cost} c>0$. The industry inverse demand function is denoted by $p(z)$ and the payoffs are $\Pi_{i}\left(q_{1}, \ldots, q_{n}\right)=\left(p\left(q_{1}+\ldots+q_{n}\right)-c\right) q_{i}$, where $q_{i}$ is the output of firm $i$.

Some reasonable assumptions about this game are:

Assumption $\mathbf{A}_{1} \cdot p: R_{+} \longrightarrow R_{+}$is continuous, differentiable and with $p^{\prime}(z)<0$ for all $z>0$ such that $p(z)>0, \lim _{z \rightarrow \infty} p(z)=0$, and $p(0)>c$.

\footnotetext{
${ }^{1}$ Since there is no danger confusion, the discount factor is not explicicitly included among its arguments.
} 
We introduce a capacity constraint in $Q_{i}$ in order to make this set compact. Formally, $Q_{i}=[0, \bar{q}(\delta)]$ for all $i=1, \ldots, n$, where $\bar{q}(\delta)$ is such that

$$
-\Pi_{1}(\bar{q}(\delta), 0, \ldots, 0)>\left(\frac{\delta}{1-\delta}\right) \sup _{q} \Pi_{1}(q, 0, \ldots, 0) .
$$

Note that this capacity constraint $\bar{q}(\delta)$ is not at all restrictive, since the loss to a firm from producing an output greater than $\bar{q}(\delta)$ cannot be recouped by any possible future gain (Abreu, 1986).

Let $q_{i}^{*}\left(q_{-i}\right)$ be a single period best response to $q_{-i}=\left(q_{1}, \ldots, q_{i-1}, q_{i+1}, \ldots, q_{n}\right)$, i.e., $q_{i}^{*}\left(q_{-i}\right)$ satisfies $\Pi_{i}\left(q_{1}, \ldots, q_{i}^{*}\left(q_{-i}\right), \ldots, q_{n}\right) \geq \Pi_{i}\left(q_{1}, \ldots, q_{i}, \ldots, q_{n}\right)$ for all $q_{i} \in$ $Q_{i}$. Set $\Pi_{i}^{*}\left(q_{1}, \ldots, q_{n}\right)=\Pi_{i}\left(q_{i}^{*}\left(q_{-i}\right), q_{-i}\right)$.

Assumption $\mathbf{A}_{2} . q_{i}^{*}\left(q_{-i}\right)$ is well defined, unique, and $q^{*}(z)=q_{1}^{*}\left(q_{2}, \ldots, q_{n}\right)$, where $z=q_{2}+\ldots+q_{n}$, is a continuous, non-increasing function.

Let $n q^{m}$ be the monopoly output level, i.e., $q^{m}$ is such that $\Pi_{1}\left(n q^{m}, 0, \ldots, 0\right) \geq$ $\Pi_{1}(q, 0, \ldots, 0)$ for all $q \in Q_{1}$.

Assumption $\mathbf{A}_{3} \cdot q^{m}$ is unique, strictly positive and $\Pi_{1}(q, \ldots, q)$ declines strictly monotonically as output increases beyond $q^{m}$ or falls below $q^{m}$.

Assumptions $\mathbf{A}_{1}, \mathbf{A}_{2}$ and $\mathbf{A}_{3}$ are equivalent to the assumptions made by Segerstrom (1988) and Abreu (1986). Segerstrom (1988) proved in his Lemma 1, that the game with two players has exactly one Cournot Nash equilibrium which is symmetric. For a game with $n$ players the generalization of this result is immediate.

We denote this unique Cournot Nash equilibrium by $\left(q^{c}, \ldots, q^{c}\right)$ and its payoff by $\Pi^{c}=\Pi_{i}\left(q^{c}, \ldots, q^{c}\right)$. Also $\left(q^{m}, \ldots, q^{m}\right)$ and $\Pi^{m}=\Pi_{i}\left(q^{m}, \ldots, q^{m}\right)$. For the sake of simplicity, we use $q^{c}$ instead of $\left(q^{c}, \ldots, q^{c}\right)$ and $q^{m}$ instead of $\left(q^{m}, \ldots, q^{m}\right)$ whenever it is clear from the context which is which.

Finally, let $\Pi^{*}(z)=\max \left\{(p(q+z)-c) q \mid q \in Q_{1}\right\}$ where $\bar{z}$ is the unique total quantity satisfying $p(\bar{z})=c$. Abreu (1986) proved that $\Pi^{*}(z)$ is strictly decreasing and convex on $[0, \bar{z}]$. 


\section{Quasi Even Stronger Perfect Equilibrium}

Simple strategy profiles are defined to avoid single player deviations (Abreu (1988)). Deviations of two or more players are always ignored. As argued in the Introduction, group deviations cannot be ignored, and any deviation by a coalition (other than the grand one) must be punished by the complementary coalition.

Now, to prevent group deviations, we introduce simple coalitional strategy profiles.

A simple coalitional strategy profile $\sigma$ is determined by $2^{n}-1$ outcome paths (one cooperative path associated with $\emptyset$ and one punishment path for each coalition other than the grand one). These outcome paths are denoted by $S^{I}$ where $S^{I}=\left\{q^{I}(t)\right\}_{t=1}^{\infty}$ for $I \subset N$, which inductively define the following strategy profile:

(i) Play $S^{\emptyset}$ until a coalition deviates singly from $S^{\emptyset}$.

(ii) For any $I \subset N$, play $S^{D}$ if coalition $D$ deviates singly from $S^{I}$, where $S^{I}$ is an ongoing previously specified path. Continue with $S^{I}$ if no further deviation occurs or if all the players deviate simultaneously.

In other words, start cooperating and continue cooperating if no player deviates. If after any history a coalition deviates, start the punishment phase of that coalition. Only deviations of all players are ignored.

Rubinstein (1980) introduced the concept of Strong Perfect Equilibrium (SPE) in repeated games. SPE requires that no coalition can improve the payoff of all of its members after any history. In view of the arguments above this is not a very strong solution concept. However in repeated games there is often no SPE. For instance Farrell (2001) proves that in the Cournot model with linear demand there is no SPE whenever $n>3$. This is because there is no way to punish the grand one. Thus, to avoid joint deviation by all the players one is restricted to using Pareto-efficient payoffs in all the punishment phases. In most cases it is not possible to deter deviations while maintaining Pareto efficiency.

So the problem of the existence of SPE is basically concerned with the grand one. This motivates the introduction of a slight modification of the concept of SPE. 
A simple coalitional strategy profile $\sigma$ is a Quasi-Strong Perfect Equilibrium (QSPE) (Horniacek (1996)) if there is no coalition $D \subset N$ and no strategy $\sigma_{D}^{\prime}$ such that for some history $h$ : $\Pi_{i}\left(\sigma_{D}^{\prime}, \sigma_{-D} \mid h\right)>\Pi_{i}(\sigma \mid h)$ for all $i \in D$.

If an equilibrium $\sigma$ is a QSPE, then the vector of payoffs $\left(\Pi_{1}(\sigma), \ldots, \Pi_{n}(\sigma)\right)$ can also be said to be a QSPE.

QSPE guarantees that no coalition (other than the grand one) will deviate in any subgame. The possibility of a deviation by the grand one is discussed below in the Conclusions.

Now it needs to be explained why it is so complex to check whether a simple coalitional strategy profile is QSPE (especially when $n$ is big). Note that deviations by any of the $2^{n}-2$ coalitions from any of the $2^{n}-1$ outcome paths need to be prevented. Each coalition could also deviate for only one period, for any finite number of periods or for ever. Furthermore, coordinated deviations $^{2}$ need to be taken into account, which could seriously complicate this problem.

The meaning of coordinated deviations here is illustrated with the following example.

Example Consider the Cournot supergame with five players, a linear demand function given by $p=100-z$ ( if $z<100$ and 0 otherwise) and a linear cost function with marginal cost $c=20$. Let $\sigma$ be the simple coalitional strategy profile defined by:

$\cdot S^{\emptyset}=\{(10,10,10,10) ;(10,10,10,10) ;(10,10,10,10) ; \ldots\}$

. $S^{\{1\}}=\{(0,50,0,0) ;(10,10,10,10) ;(10,10,10,10) ; \ldots\}$.

. $S^{\{2\}}=\{(50,0,0,0) ;(10,10,10,10) ;(10,10,10,10) ; \ldots\}$.

. $S^{\{3\}}=\{(0,0,0,50) ;(10,10,10,10) ;(10,10,10,10) ; \ldots\}$.

. $S^{\{4\}}=\{(0,0,50,0) ;(10,10,10,10) ;(10,10,10,10) ; \ldots\}$.

. $S^{\{1,2\}}=\{(0,0,25,25) ;(10,10,10,10) ;(10,10,10,10) ; \ldots\}$ and for $\{i, j\} \neq$ $\{1,2\}, S^{\{i, j\}}$ is identical to $S^{\{1,2\}}$ except that the roles of players 1 and 2 , and players $i$ and $j$ are interchanged.

$\cdot S^{\{1,2,3\}}=\{(0,0,0,50) ;(10,10,10,10) ;(10,10,10,10) ; \ldots\}$ and for $\{i, j, k\} \neq$

\footnotetext{
${ }^{2}$ The problem of these coordinated deviations was pointed out by Rubinstein (1980). In his paper he called them "deviations by stages".
} 
$\{1,2,3\}, S^{\{i, j, k\}}$ is identical to $S^{\{1,2,3\}}$ except that the roles of players 1,2 and 3 , and players $i, j$ and $k$ are interchanged.

In this example it can be shown by simple but rather long computations, that if $\delta>0.5625$ no coalition $D \subset N$ can obtain more benefits for all of its members with joint deviations of $\sigma$. However, players 1 and 2 can obtain more benefits by coordinating their deviations according to the following plan: First player 2 deviates singly from $S^{\{1\}}$. In the next period $\sigma$ recommends that $S^{\{2\}}$ be started. Instead, player 1 deviates from $S^{\{2\}}$, while player 2 follows $\sigma$. Then $\sigma$ recommends that $S^{\{1\}}$ be started again. But then again player 2 deviates singly from $S^{\{1\}}$, and in the next period player 1 deviates from $S^{\{2\}}$, and so on. Note that $\Pi^{m}=\Pi_{1}(10,10,10,10)=\Pi_{2}(10,10,10,10)=400$, $q_{1}^{*}(0,0,0)=q_{2}^{*}(0,0,0)=40, \Pi_{1}^{*}(40,0,0,0)=\Pi_{2}^{*}(0,40,0,0)=1600$ and $\Pi_{1}(50,0,0,0)=\Pi_{2}(0,50,0,0)=1500$. Then, for $\delta>0.333$, the result for player 1 is: $1600+0 \delta+1600 \delta^{2}+0 \delta^{3}+\ldots>1500+400 \delta+400 \delta^{2}+400 \delta^{3}+\ldots$ and for player 2 : $0+1600 \delta+0 \delta^{2}+1600 \delta^{3}+\ldots>0+400 \delta+400 \delta^{2}+400 \delta^{3}+\ldots$ So both players find it more profitable to follow the above plan of alternating deviations than to continue with $\sigma$. The conclusion is that although coalition $\{1,2\}$ is not able to obtain more benefits for all of its members with a joint deviation from $\sigma$ (after any history), players 1 and 2 are able to improve their payoffs by coordinating their actions.

In general a coordinated deviation by a coalition $D$ alternates deviations by subcoalitions $B \subset D$ according to a plan which could, potentially, be infinitely complex. This seriously complicates the problem of checking that no coalition $D \subset N$ will ever deviate. In order to deal with this problem a new equilibrium concept which is even stronger than SPE needs to be introduced.

Definition A simple coalitional strategy profile $\sigma$ is a Quasi-Even Stronger Perfect Equilibrium (QESPE ) if there is no coalition $D \subset N$ and no strategy $\sigma_{D}^{\prime}$ such that for some history $h: \sum_{i \in D} \Pi_{i}\left(\sigma_{D}^{\prime}, \sigma_{-D} \mid h\right)>\sum_{i \in D} \Pi_{i}(\sigma \mid h)$.

If an equilibrium $\sigma$ is a QESPE, then the vector of payoffs $\left(\Pi_{1}(\sigma), \ldots, \Pi_{n}(\sigma)\right)$ can also be said to be a QESPE. 
QESPE is just an intermediate concept. We do not claim that it is interesting in itself. We use it to prove the existence of QSPE. Note that if $\sigma$ is QESPE then $\sigma$ is also SPE, whereas the reverse is not true.

This new concept allows us to obtain a result that significantly simplifies the process of checking that no coalition will ever deviate. Abreu (1988) proves that simple strategy profiles are simple not only because of their definition but also because it is easy to check whether they are subgame perfect equilibria. According to his Proposition 1 (p. 391), only one-shot deviations need be checked to ensure subgame perfection, where a one-shot deviation from a strategy consists of a single period deviation followed by sticking to the strategy afterwards. The proposition below generalizes this finding by Abreu to the QESPE concept.

Proposition 1 The simple coalitional strategy profile $\sigma$ is a QESPE if and only if $\sum_{i \in D} \Pi_{i}\left(q_{B}, q_{-B}^{I}(t)\right)+\delta \sum_{i \in D} \Pi_{i}\left(S^{B}\right) \leq \sum_{i \in D} \Pi_{i}\left(S^{I}, t\right)$ for all $q_{B} \in Q_{B}$, $B \subseteq D, D \subset N, I \subset N$ and $t=1,2, \ldots$

This condition means that no coalition can in sum obtain more payoffs with a single period joint deviation followed by sticking to the strategy thereafter. Nor are one-shot coordinated deviations profitable in sum for any subcoalition $B \subset D$.

\section{QESPE in the Cournot Supergame}

Proposition in Horniacek (1996) establishes a sufficient condition for an action vector to be sustained by a QSPE (for a discount factor close enough to 1). From this condition it is straightforward to prove the existence of QSPE in the Cournot model. However this result does not guarantee the Pareto optimality of the payoffs.

In this section we prove that, under assumptions A1-A3, the symmetric monopoly outcome can be sustained as a QSPE even when the number of players tends to infinity.

Next we given sufficient conditions to sustain the symmetric monopoly outcome as a QESPE. 
Proposition 2 The symmetric monopoly payoff vector $\left(\Pi^{m}, \ldots, \Pi^{m}\right)$ is QESPE for $\delta$ close enough to 1 if there exists an action profile $q^{p}=\left(q^{\prime}, q, \ldots q\right) \in Q$ such that:

(i) $\Pi_{1}\left(q^{p}\right) \leq \Pi_{2}\left(q^{p}\right) \leq \Pi^{m}$.

(ii) $\Pi_{1}^{*}\left(q^{p}\right)<\Pi^{m}$.

Note that when $\Pi_{2}\left(q^{p}\right) \leq \Pi^{m}$ the problem of coordinated deviations does not arise and everything is much simpler. Aramendia et al. (2002) prove in their Theorem 6 that in the Cournot model with standard assumptions there is always $\bar{n}$ such that for all $n \geq \bar{n}$ it is not possible to sustain $\Pi^{m}$ (with a simple, two-phase strategy profile) unless $\Pi_{2}\left(q^{p}\right) \leq \Pi^{m}$. Hence, although this condition seems to be very strong, it is in fact necessary to sustain $\Pi^{m}$ when there is a high enough number of players (9 in the linear case).

The simple coalitional strategy profile $\sigma$ is constructed as follows: Let $q^{p}=\left(q^{\prime}, q, \ldots q\right)$ be the vector of quantities that it is recommended to play in the first period of $S^{1}$ of the two-phase subgame perfect equilibrium. The corresponding QESPE strategy profile $\sigma$ is defined by $S^{\emptyset}=\left\{q^{m}\right\}_{t=1}^{\infty}$ and $S^{I}=\left\{q^{I}, \ldots, q^{I}, q^{m}, q^{m}, \ldots\right\}$ for $I \subset N$, where $q_{i}^{I}=\frac{q^{\prime}+(|I|-1) q}{|I|}$ if $i \in I$ and $q_{i}^{I}=q$ otherwise. See the proof of Proposition 2 to understand why this procedure works. The number of periods in the punishment phase of coalitions $I$ with more than one player may have to be increased, and some margin will probably also be lost in the discount factor, but anyway the existence of QESPE (and therefore of QSPE) is guaranteed for $\delta$ close enough to 1

Let $\sigma$ be a two-phase simple strategy profile such that $S^{\emptyset}=\left\{q^{m}\right\}_{t=1}^{\infty}, S^{1}=$ $\left\{q^{p}, \ldots, q^{p}, q^{m}, q^{m}, \ldots\right\}$ and, for $i \neq 1, S^{i}$ is identical to $S^{1}$ except that the roles of players 1 and $i$ are interchanged. Note that if a strategy of this kind is a subgame perfect equilibrium and satisfies the requirement that $\Pi_{2}\left(q^{p}\right) \leq \Pi^{m}$, then conditions (i) and (ii) of the above Proposition 2 are automatically met. This is because $\Pi_{1}\left(q^{p}\right) \leq \Pi^{m}$ and condition (ii) is necessary to ensure subgame perfection.

The conclusion is surprising:

Corollary If the symmetric monopoly outcome can be sustained as a subgame perfect equilibrium with a two-phase simple strategy profile satisfying $\Pi_{2}\left(q^{p}\right) \leq \Pi^{m}$, then there is a simple, two-phase coalitional strategy profile that 
also sustains the symmetric monopoly outcome as a QESPE (and therefore as a $Q S P E)$..

Now it is straightforward to prove the central theorem of this paper.

Theorem The symmetric monopoly outcome can be sustained as a QESPE (and therefore as a QSPE) for any number of players provided that the discount factor is close enough to 1.

The simple coalitional strategy profile strategy $\sigma$ considered in the proof of this theorem is defined by $S^{\emptyset}=\left\{q^{m}\right\}_{t=1}^{\infty}$ and $S^{I}=\left\{q^{c}, \ldots, q^{c}, q^{m}, q^{m}, \ldots\right\}$ for $I \subset N$. This strategy is not the trigger strategy although it is very similar. Note that playing $q^{c}$ forever, i.e., $S^{I}=\left\{q^{c}, q^{c}, q^{c}, \ldots\right\}$ is not QSPE since any coalition $D$ with more than one player can deviate from $q^{c}$ in any period.

The next step is to show that there is a variety of QESPE strategies for which the symmetric monopoly outcome can be sustained, and that some of these strategies may have a very different structure. To show this we include two different types of QESPE strategy:

Type 1. Let $\sigma$ be defined by $S^{\emptyset}=\left\{q^{m}\right\}_{t=1}^{\infty}$ and $S^{I}=\left\{q^{p}, \ldots, q^{p}, q^{m}, q^{m}, \ldots\right\}$ for $I \subset N$, where $q^{p}=\left(q^{\prime}, \ldots, q^{\prime}\right)$ is such that $p\left((n-1) q^{\prime}\right) \leq c$.

To see that $\sigma$ is QESPE it suffices to check that conditions (i) and (ii) of Proposition 2 are satisfied. As $\Pi_{1}\left(q^{p}\right)=\Pi_{2}\left(q^{p}\right)<0$ and $\Pi_{1}^{*}\left(q^{p}\right)=0$ the result follows. Therefore all these strategies sustain $\left(\Pi^{m}, \ldots, \Pi^{m}\right)$ as a QESPE for $\delta$ close enough to 1 . Note that strategies of this kind have a stick and carrot structure and are similar to the strategies proposed by Abreu (1986). However two differences should be pointed out: the first is that $t$ periods of punishment may be needed instead of only one. The second is that in the carrot phase of the punishment we always come back to $q^{m}$ whereas Abreu, looking for the most severe punishment, returns in most cases to a level with less profits than $\Pi^{m}$.

Now we propose a new type of strategy that also sustains the monopoly outcome as an QESPE. The idea is to force the cheater to accept the punishment, thus ensuring that this player in question will not deviate during his punishment phase. 
Type 2. Let $\sigma$ be given by $S^{\emptyset}=\left\{\left(q^{m}, \ldots, q^{m}\right) ;\left(q^{m}, \ldots, q^{m}\right) ; \ldots\right\}$ and $S^{I}=$ $\left\{q^{I}, \ldots, q^{I}, q^{m}, q^{m}, \ldots\right\}$ for $I \subset N$, where $q_{i}^{I}=\frac{R(q)+(|I|-1) q}{|I|}$ if $i \in I$ and $q_{i}^{I}=q$ otherwise, whith $R(q)=q^{*}((n-1) q)$.

This strategy sustain $\left(\Pi^{m}, \ldots, \Pi^{m}\right)$ as QESPE (when $\delta$ is big enough) for all $q \in\left[q^{c}, \frac{\bar{z}}{n-1}\right]$ such that $\Pi_{2}(R(q), q, \ldots, q) \leq \Pi^{m}$. We show that conditions (i) and (ii) of Proposition 2 hold. Given that $R(q)$ is non increasing (by assumption $\left.A_{2}\right)$, then $R(q) \leq R\left(q^{c}\right)=q^{c}$ for all $q \in\left[q^{c}, \frac{\bar{z}}{n-1}\right]$. Thus $R(q) \leq q$ and $\Pi_{1}(R(q), q, \ldots, q) \leq \Pi_{2}(R(q), q, \ldots, q) \leq \Pi^{m}$, hence condition (i) follows. Moreover, as $\Pi^{*}(z)$ is decreasing on $[0, \bar{z}]$ we have $\Pi_{1}^{*}(R(q), q, \ldots, q)=\Pi^{*}((n-$ 1) $q) \leq \Pi^{*}\left((n-1) q^{c}\right)=\Pi^{c}<\Pi^{m}$. Hence (ii) holds.

In the linear Cournot model, it can be easily shown that $\Pi_{2}(R(q), q, \ldots, q) \leq$ $\Pi^{m}$ for all $q \in\left[q^{c}, \frac{\bar{z}}{n-1}\right]$, so we conclude that $\sigma$ is QESPE for all $q \in\left[q^{c}, \frac{\bar{z}}{n-1}\right]$.

\section{Conclusions}

In this paper we show that, in repeated games, coalitional deviations may have to be punished. Otherwise coalitions could sustain their deviations with QSPE strategies (i.e., strategies that meet the condition that no subcoalition deviates further). In this setting, the subgame perfect equilibrium is therefore a weak equilibrium concept. This conclusion is not new. It was reached by Horniacek with a different, original argument.

One question that remains outstanding is this: what about deviations by the grand one?

To prevent deviations by the grand one one would have to choose the optimal strategy among all the QSPE strategies according to a criterion. Using a common expression in game theory, it can be said that a QSPE strategy is a strategy with internal stability. In these terms external stability means that there is no other strategy which is QSPE and is preferred by all players. As shown above, in the Cournot model, there are infinite strategies that are QSPE. However, due to the complexity of the problem, it is not vet possible to characterize all QSPE strategies. We are only able to present results concerning existence. That is why, this paper, does not attempt to establish the external stability condition.

Horniacek (1996) seeks to approximate a Strong Perfect Equilibrium in 
the setting of discounted supergames. He solves the problem of deviations by the grand one by imposing two additional requirements on the QSPE ${ }^{3}$ concept. The first is that no coalition can increase the average payoff of at least one of its members by more than $\epsilon$ without decreasing the average payoff of any other members. The second is to add Weak Renegotiation Proofness in the sense of Farrell and Maskin (1989). Horniacek proves necessary and sufficient conditions for the existence of this concept. However the strategies used in his proofs are really complex. We believe that the main contribution of our work is to simplify this problem by means of simple coalitional strategy profiles. We believe it might be of interest to follow Horniacek's approach using our results to advance in his line of investigation.

\section{Appendix}

Proof. (Proposition 1) We must show that $\sigma$ is a QESPE if and only if

$$
\sum_{i \in D} \Pi_{i}\left(q_{B}, q_{-B}^{I}(t)\right)+\delta \sum_{i \in D} \Pi_{i}\left(S^{B}\right) \leq \sum_{i \in D} \Pi_{i}\left(S^{I}, t\right)
$$

for all $q_{B} \in Q_{B}, B \subseteq D, D \subset N, I \subset N$ and $t=1,2, \ldots$.

Following the same steps as Abreu in his Proposition 1, from the point of view of coalition $D, \sigma_{-D}$ defines a stationary discounted Markov decision problem with states $q_{-D}^{I}(t), I \subset N, t=1,2, \ldots$. Transition probabilities are given by $\operatorname{prob}\left(q_{-D}^{I}(t+1) \mid q_{-D}^{I}(t), q_{D}\right)=1$ if $q_{D}=q_{D}^{I}(t)$ and $\operatorname{prob}\left(q_{-D}^{B}(1) \mid q_{-D}^{I}(t), q_{D}\right)=1$ if $q_{B} \neq q_{B}^{I}(t)$. The payoff of coalition $D$ in each period for each state is $g\left(q_{-D}^{I}(t), q_{D}\right)=\sum_{i \in D} \Pi_{i}\left(q_{D}, q_{-D}^{I}(t)\right)$. Then, by Proposition 7, Chapter 6, Bertsekas (1976) the inequalities (1) assert that $\sigma_{D}$ is optimal, i.e., $\left(\sigma_{D} \mid h\right)$ is a best response to $\left(\sigma_{-D} \mid h\right)$ for all history $h$. This establishes sufficiency. Necessity follows directly from the definition of QESPE.

Let $\sigma$ be a simple coalitional strategy profile with a two-phase punishment such that $S^{\emptyset}=\left\{q^{m}\right\}_{t=1}^{\infty}$ and $S^{I}=\left\{q^{I}, \ldots, q^{I}, q^{m}, \ldots\right\}$ for $I \subset N$, where the cooperative path $S^{\emptyset}$ takes the action $q^{m}$ for each period and the punishment path $S^{I}$ takes the action $q^{I}, t$ times, and then $q^{m}$ forever. The

\footnotetext{
${ }^{3}$ To be precise we have to say that he works with an equilibrium concept which is very close to QSPE. He calls it Semi Strict Quasy Strong Perfect Equilibrium.
} 
following lemma establishes sufficient conditions for a strategy of this kind to be QESPE provided that the players are sufficiently patient.

First we introduce some useful notation. Let $D$ be a coalition of $N$. Set $q_{D}^{*}\left(q_{-D}\right)$ such that $\sum_{i \in D} \Pi_{i}\left(q_{D}^{*}\left(q_{-D}\right), q_{-D}\right) \geq \sum_{i \in D} \Pi_{i}\left(q_{D}, q_{-D}\right)$ for all $q_{D} \in Q_{D}$ and $\Pi_{D}^{*}\left(q_{1}, \ldots, q_{n}\right)=\sum_{i \in D} \Pi_{i}\left(q_{D}^{*}\left(q_{-D}\right), q_{-D}\right)$.

Lemma 1 A simple coalitional strategy profile with a two-phase punishment $\sigma$ is QESPE for a suitable $t$ and $\delta$ close enough to 1 if the following conditions hold:

(a) $\sum_{i \in D} \Pi_{i}\left(q^{B}\right)<|D| \Pi^{m}$ for all $B \subseteq D, D \subset N$.

(b) $\sum_{i \in D} \Pi_{i}\left(q^{B}\right) \leq \sum_{i \in D} \Pi_{i}\left(q^{I}\right)$ for all $B \subseteq D$ and $D, I \subset N$.

(c) If $\sum_{i \in D} \Pi_{i}\left(q^{B}\right)=\sum_{i \in D} \Pi_{i}\left(q^{I}\right)$ for some $B \subseteq D$ and $D, I \subset N$ then $\sum_{i \in D} \Pi_{i}\left(q^{*}\left(q_{-B}^{I}\right), q_{-B}^{I}\right)<$ $|D| \Pi^{m}$.

Proof. By Proposition 1 only one-shot deviations need be checked to ensure that $\sigma$ is a QESPE. In this case only the following one-shot deviations need be checked:

(i) Deviations of coalition $D$ from path $S^{\emptyset}$. To avoid this we must show that the following inequality hold:

$$
\sum_{i \in D} \Pi_{i}\left(q^{*}\left(q_{-B}^{m}\right), q_{-B}^{m}\right)+\delta \sum_{i \in D} \Pi_{i}\left(S^{B}\right) \leq \sum_{i \in D} \Pi_{i}\left(S^{\emptyset}\right) .
$$

Let $F(\delta)=\sum_{i \in D} \Pi_{i}\left(q^{*}\left(q_{-B}^{m}\right), q_{-B}^{m}\right)+\delta \sum_{i \in D} \Pi_{i}\left(S^{B}\right)-\sum_{i \in D} \Pi_{i}\left(S^{\emptyset}\right)$. As $\Pi_{i}\left(S^{B}\right)=$ $\Pi_{i}\left(q^{B}\right)+\delta \Pi_{i}\left(q^{B}\right)+\ldots+\delta^{t} \Pi_{i}\left(q^{B}\right)+\delta^{t+1} \Pi^{m}+\ldots$ and $\Pi_{i}\left(S^{\emptyset}\right)=\Pi^{m}+\delta \Pi^{m}+$ $\ldots+\delta^{t} \Pi^{m}+\delta^{t+1} \Pi^{m}+\ldots$ then $F(\delta)=\sum_{i \in D} \Pi_{i}\left(q^{*}\left(q_{-B}^{m}\right), q_{-B}^{m}\right)-|D| \Pi^{m}-(\delta+$ $\left.\left.\ldots+\delta^{t}\right)\left(|D| \Pi^{m}\right)-\sum_{i \in D} \Pi_{i}\left(q^{B}\right)\right)$. Therefore, $\lim _{\delta \rightarrow 1} F(\delta)=\sum_{i \in D} \Pi_{i}\left(q^{*}\left(q_{-B}^{m}\right), q_{-B}^{m}\right)-$ $|D| \Pi^{m}-t\left(|D| \Pi^{m}-\sum_{i \in D} \Pi_{i}\left(q^{B}\right)\right)$.

Then, taking $t>\frac{\sum_{i \in D} \Pi_{i}\left(q^{*}\left(q_{-B}^{m}\right), q_{-B}^{m}\right)-|D| \Pi^{m}}{|D| \Pi^{m}-\sum_{i \in D} \Pi_{i}\left(q^{B}\right)}$, by condition (a), we have that $\lim _{\delta \rightarrow 1} F(\delta)<0$ and $(2)$ holds for $\delta$ close enough to 1 .

(ii) Deviations by coalition $D$ from $S^{I}$ in the first period. Now the inequality to be checked is:

$$
\sum_{i \in D} \Pi_{i}\left(q^{*}\left(q_{-B}^{I}\right), q_{-B}^{I}\right)+\delta \sum_{i \in D} \Pi_{i}\left(S^{B}\right) \leq \sum_{i \in D} \Pi_{i}\left(S^{I}\right) .
$$


Let $F(\delta)=\sum_{i \in D} \Pi_{i}\left(q^{*}\left(q_{-B}^{I}\right), q_{-B}^{I}\right)+\delta \sum_{i \in D} \Pi_{i}\left(S^{B}\right)-\sum_{i \in D} \Pi_{i}\left(S^{I}\right)$.Then $F(\delta)=$ $\sum_{i \in D} \Pi_{i}\left(q^{*}\left(q_{-B}^{I}\right), q_{-B}^{I}\right)-|D| \Pi^{m}-\left(1+\delta+\ldots+\delta^{t-1}\right) \sum_{i \in D}\left(\Pi_{i}\left(q^{I}\right)-\Pi_{i}\left(q^{B}\right)\right)+$ $\left(1-\delta^{t}\right)\left(|D| \Pi^{m}-\sum_{i \in D}\left(\Pi_{i}\left(q^{B}\right)\right)\right.$.

Hence $\lim _{\delta \rightarrow 1} F(\delta)=\sum_{i \in D} \Pi_{i}\left(q^{*}\left(q_{-B}^{I}\right), q_{-B}^{I}\right)-|D| \Pi^{m}-t \sum_{i \in D}\left(\Pi_{i}\left(q^{I}\right)-\Pi_{i}\left(q^{B}\right)\right)$. Now, by condition (b), $\sum_{i \in D} \Pi_{i}\left(q^{B}\right) \leq \sum_{i \in D}\left(\Pi_{i}\left(q^{I}\right)\right.$. Therefore, if $\sum_{i \in D} \Pi_{i}\left(q^{B}\right)<$ $\sum_{i \in D}\left(\Pi_{i}\left(q^{I}\right)\right.$, taking $t>\frac{\sum_{i \in D} \Pi_{i}\left(q^{*}\left(q_{-B}^{I}\right), q_{-B}^{I}\right)-|D| \Pi^{m}}{\sum_{i \in D}\left(\Pi_{i}\left(q^{I}\right)-\Pi_{i}\left(q^{B}\right)\right)}$, we have that $\lim _{\delta \rightarrow 1} F(\delta)<0$.

Clearly if $\sum_{i \in D} \Pi_{i}\left(q^{B}\right)=\sum_{i \in D}\left(\Pi_{i}\left(q^{I}\right)\right.$, by $(\mathrm{c}), \lim _{\delta \rightarrow 1} F(\delta)=\sum_{i \in D} \Pi_{i}\left(q^{*}\left(q_{-B}^{I}\right), q_{-B}^{I}\right)-$ $|D| \Pi^{m}<0$. So (3) is true for $\delta$ close enough to 1 .

Finally it is straightforward that if a deviation from $q^{I}$ in the first period of $S^{I}$ is not profitable then a deviation from $q^{I}$ in periods $2, \ldots, t$ is not profitable either.

Note that when $B=D=I$, condition (c) of this lemma is simply $\Pi_{D}^{*}\left(q^{D}\right)<|D| \Pi^{m}$. This condition must be met because otherwise coalition $D$ will first deviate from $S^{\emptyset}$ and then play $q_{D}^{*}\left(q^{D}\right)$ forever. The following lemma makes this condition easier to work with.

Lemma 2 If $\Pi_{1}^{*}(q, \ldots, q)<\Pi^{m}$ then $\Pi_{D}^{*}(q, \ldots, q)<|D| \Pi^{m}$ for all $D \subset N$.

Proof. We first prove the following assertion ${ }^{4}$ :

$$
\text { if } \Pi^{*}(n q) \leq \Pi_{n}^{m} \text { then } \Pi^{*}((n-k) q) \leq k \Pi_{n}^{m} \text { for all } k=2, \ldots, n
$$

We argue by induction on $n$. If $n=2$ then $\Pi^{*}((n-k) q)=\Pi^{*}(0)=2 \Pi_{2}^{m}$ and the result follows. For $n \geq 2$, if $k=n$ we have $\Pi^{*}((n-k) q)=\Pi^{*}(0)=n \Pi_{n}^{m}$. Suppose now that $k \leq n-1$. Set $q^{\prime}=\left(\frac{n}{n-1}\right) q$. Then $(n-k) q=(n-$ k) $\left(\frac{n-1}{n}\right) q^{\prime}=\left(1-\frac{k}{n}\right)(n-1-k) q^{\prime}+\frac{k}{n}((n-1)-(k-1)) q^{\prime}$. As $\Pi^{*}(z)$ is convex in $[0, \bar{z}]$ we have $\Pi^{*}((n-k) q) \leq\left(1-\frac{k}{n}\right) \Pi^{*}\left((n-1-k) q^{\prime}\right)+\frac{k}{n} \Pi^{*}((n-1-(k-$ 1)) $\left.q^{\prime}\right)$. Given that $\Pi^{*}\left((n-1) q^{\prime}\right)=\Pi^{*}(n q) \leq \Pi_{n}^{m}=\frac{n-1}{n} \Pi_{n-1}^{m}<\Pi_{n-1}^{m}$, by the inductive hypothesis, we have $\Pi^{*}((n-k) q) \leq\left(1-\frac{k}{n}\right) k \Pi_{n-1}^{m}+\frac{k}{n}(k-1) \Pi_{n-1}^{m}=$ $k \frac{n-1}{n} \Pi_{n-1}^{m}=k \Pi_{n}^{m}$ and (4) holds.

\footnotetext{
${ }^{4}$ We write $\Pi_{n}^{m}$ instead of $\Pi^{m}$.
} 
Now, as $\Pi^{*}(z)$ is strictly decreasing on $[0, \bar{z}]$ the equation $\Pi^{*}((n-k) q)=$ $k \Pi_{n}^{m}$ has a unique solution $\bar{q}_{k}$ and the following assertion holds:

$$
\Pi^{*}((n-k) q)<k \Pi_{n}^{m} \text { if and only if } q>\bar{q}_{k} .
$$

Therefore, if $\Pi^{*}((n-1) q)<\Pi_{n}^{m}$ then $q>\bar{q}_{1}$. As $\Pi^{*}\left(n \bar{q}_{1}\right)<\Pi^{*}\left((n-1) \bar{q}_{1}\right)=$ $\Pi_{n}^{m}$, by (4), we have $\Pi^{*}\left((n-k) \bar{q}_{k}\right) \leq k \Pi_{n}^{m}$, and by $(5), \bar{q}_{1} \geq \bar{q}_{k}$. Hence $q>\bar{q}_{k}$ and $\Pi^{*}((n-k) q)<k \Pi_{n}^{m}$. It follows that if $\Pi^{*}((n-1) q)<\Pi_{n}^{m}$ then $\Pi^{*}((n-k) q)<k \Pi_{n}^{m}$ for all $k=2, \ldots, n$.

Finally the result follows taking into account that $\Pi_{1}^{*}(q, \ldots, q)=\Pi^{*}((n-$ 1)q) and $\Pi_{D}^{*}(q, \ldots, q)=\Pi^{*}((n-|D|) q)$ for all $D \subset N$.

Proof. (Proposition 2) Consider the simple coalitional strategy profile $\sigma$ defined by $S^{\emptyset}=\left\{q^{m}\right\}_{t=1}^{\infty}$.and $S^{I}=\left\{q^{I}, \ldots, q^{I}, q^{m}, q^{m}, \ldots\right\}$ for $I \subset N$, where $q_{i}^{I}=\frac{q^{\prime}+(|I|-1) q}{|I|}$ if $i \in I$ and $q_{i}^{I}=q$ otherwise. We prove that the conditions of Lemma 1 are satisfied. By construction, $\Pi_{i}\left(q^{I}\right)=\frac{\Pi_{1}\left(q^{p}\right)+(|I|-1) \Pi_{i}\left(q^{p}\right)}{|I|}$ if $i \in I$ and $\Pi_{i}\left(q^{I}\right)=\Pi_{i}\left(q^{p}\right)$ otherwise. Then, for $B \subseteq D$, by (i) and (ii), we have that $\sum_{i \in D} \Pi_{i}\left(q^{B}\right)=\Pi_{1}\left(q^{p}\right)+(|B|-1) \Pi_{i}\left(q^{p}\right)+(|D|-|B|) \Pi_{i}\left(q^{p}\right)=\Pi_{1}\left(q^{p}\right)+$ $(|D|-1) \Pi_{i}\left(q^{p}\right)<|D| \Pi^{m}$. Hence, condition (a) of Lemma 1 is satisfied. Now, we show that condition (b) holds. We have $\sum_{i \in D} \Pi_{i}\left(q^{I}\right)-\sum_{i \in D} \Pi_{i}\left(q^{B}\right)=$ $|D \cap I| \frac{\Pi_{1}\left(q^{p}\right)+(|I|-1) \Pi_{i}\left(q^{p}\right)}{|I|}+|D \backslash I| \Pi_{i}\left(q^{p}\right)-\Pi_{1}\left(q^{p}\right)+(|D|-1) \Pi_{i}\left(q^{p}\right)$. Then $\sum_{i \in D} \Pi_{i}\left(q^{I}\right)-\sum_{i \in D} \Pi_{i}\left(q^{B}\right)=(|I|-|D \cap I|)\left(\Pi_{i}\left(q^{p}\right)-\Pi_{1}\left(q^{p}\right)\right)$ and by $(\mathrm{i}), \sum_{i \in D} \Pi_{i}\left(q^{I}\right)-$ $\sum_{i \in D} \Pi_{i}\left(q^{B}\right) \geq 0$. Furthermore, the equality holds if and only if $I \subseteq D$ or $q^{\prime}=q$. In this case, $\sum_{i \in D} \Pi_{i}\left(q^{*}\left(q_{-B}^{I}\right), q_{-B}^{I}\right) \leq \Pi_{D}^{*}\left(q^{I}\right)=\Pi^{*}((n-|D|) q)=\Pi_{D}^{*}(q)$. Given that, by (ii), $\Pi_{1}^{*}(q)=\Pi_{1}^{*}\left(q^{p}\right)<\Pi^{m}$, then by Lemma $2, \Pi_{D}^{*}(q)<|D| \Pi^{m}$ and therefore condition (c) is also satisfied.

Proof. (Theorem) It suffices to show the existence of a quantity vector $q^{p}=\left(q^{\prime}, q, \ldots q\right)$ such that the sufficient conditions of Proposition 2 hold. The possibilities are infinite. The simplest one is to take $q^{p}=q^{c}$. Then $\Pi_{1}\left(q^{c}\right)=\Pi_{2}\left(q^{c}\right)=\Pi^{c}$ and $\Pi_{1}^{*}\left(q^{c}\right)=\Pi^{c}$. As $\Pi^{c}<\Pi^{m}$ the conditions of Proposition 2 are satisfied and the result follows. 


\section{References}

[1] D. Abreu, (1986) Extremal Equilibria of Oligopolistic Supergames, Journal of Economic Theory 39, 191-225.

[2] D. Abreu, (1988) On the Theory of Infinitely Repeated Games with Discounting, Econometrica 56, 383-396.

[3] M. Aramendía, C. Larrea and L. Ruiz, (2005) Renegotiation in the repeated Cournot model. Games and Economic Behavior 52, 1-19.

[4] D. Bertsekas, (1976) Dynamic Programming and Stochastic Control. New York: Academic Press.

[5] J. Farrell (2000) Renegotiation in Repeated Oligopoly Interaction, Incentives, Organization and Public Economies: Papers in Honour of Sir James Mirrlees, ed. G. Myles and P. Hammond. Oxford University Press.

[6] J. Farrell, and E. Maskin, (1989) Renegotiation in Repeated Games. Games and Economic Behavior 1, 327-360.

[7] M. Horniacek, (1996) The Approximation of a Strong Perfect Equilibrium in a Discounted Supergame, Journal of Mathemathical Economics 25, 85107.

[8] A. Rubinstein, (1980) Strong Perfect Equilibrium in Supergames, International Journal of Game Theory 9, 1-12.

[9] P.S. Segestrom, (1988) Demons and Repentance, Journal of Economic Theory 45, 32-52. 